\title{
Rapid Sizing Tool for a Low Cost University Microsatellite Structure Subsystem
}

\author{
Ali Ravanbakhsh and Sebastian Franchini \\ Instituto Universitario "Ignacio Da Riva" (IDR/UPM) \\ E.T.S.I. Aeronáuticos, Universidad Politécnica de Madrid \\ Madrid, Spain \\ ali.ravanbakhsh@upm.es
}

\begin{abstract}
In recent decays university class small satellites are creating many opportunities for space research and professional trainings while at the same time responding to constrained budgets. In this work the main focus is on developing a simple and rapid structural sizing tool considering the main objectives of a low cost university class microsatellite project. In satellite projects, structure subsystem is one of the influential subsystems as a driver of the cost and acceptance of the final design. At the first steps of such projects there is no confirmed data regarding the launch vehicle or even in some cases there is no data for the satellite payload. Due to these facts, developing simple sizing tools at conceptual design phase for obtaining an over view of the effect of different variables is useful before entering complex calculations in detailed design phases. In this study, after developing a simple analytical model of satellite structure subsystem, a design space is evaluated with practical boundaries considering mass and dimensions constraints of such projects. The results are useful to give initial insight to establish the system level structural sizing.
\end{abstract}

\section{INTRODUCTION}

For more than two decades small satellites have been among promising choices for universities and research institutes for initiation of space technology development programs in an academic environment. According to online resources, from 1981, the launch date of the first university microsatellite named UoSAT-1 developed by University of Surrey [1], until 2008 more than 100 satellite projects have been conducted at universities. And, more than $70 \%$ of these projects are included in microsatellite class whose weight is between 20-70 kg [2]. Satellite design at any class is a complex, iterative process that involves multi-disciplinary engineering expertise. This inherent complexity exists also in the case of a university microsatellite project whose main purpose is educational training and technology demonstration without any top level stakeholder's requirements. Due to this fact, developing rapid sizing tools for design driver disciplines is really useful. This will provide the design team an insight toward the system design which is mandatory before stepping in detailed design in such complex projects. In the present work the main focus is on developing a rapid and simple sizing tool for structure subsystem. Structure subsystem, especially in case of microsatellites plays an important role in final mass and also cost of the project. On the other hand based of uncertainties about the launch of a university class small satellites, the structural design should be done under special concerns. Satellite dimensions and stiffness can be considered as the most important requirements for these satellite projects owing to the fact that they are intended to be launched as secondary payload for project cost reduction. In the following sections, after assuming a common geometrical architecture for satellite, a simple structural model is developed and the effect of satellite total mass and its dimensions on some design indicators are investigated.

\section{SATELlite ARCHITECTURE}

\section{A. Primary structures}

According to [3] the box configuration is the most common configuration for small satellites, also considering different university microsatellite projects $[4][5][6][7][8]$, it seems that the cubic based parallelized sides box is the most appropriate geometry architecture for these kinds of projects. On the other hand this configuration can be achieved by simple structural elements like beams, cleats and plates. This simplicity in structural elements will decrease the production cost which is a great advantage in most of the university class microsatellite projects. The satellite primary structures are generally designed by considering stiffness, or natural frequency, and to endure steady state accelerations and transient loading during launch period. The primary structural elements have the most mass budget among structure mass budget in comparison with 
secondary and tertiary structural elements and the whole satellite structure design in early stages can be based on primary structures design. In this paper, in order to have a low cost structure subsystem, the primary structure elements material are considered to be normal isotropic material, Al7075. Structural elements consist of four equal leg angle beams for satellite main frame, four normal plates as satellite side panels and four isogrid plates acting as satellite middle trays. In middle trays structural design, it is planned to use isogrid structure plates instead of honeycombs or normal plates. This is because of their less mass in comparison to monocoque plates and less cost and complexity in manufacturing compared to honeycombs. The sizing of isogrid plates is done by design relationships from [9] while fulfilling the frequency requirements using square plates natural frequencies from [10]. Although isogrid plates are not as efficient as honeycombs, they are promising for these kinds of projects [11] and [12]. The primary structures are shown in Fig.1.

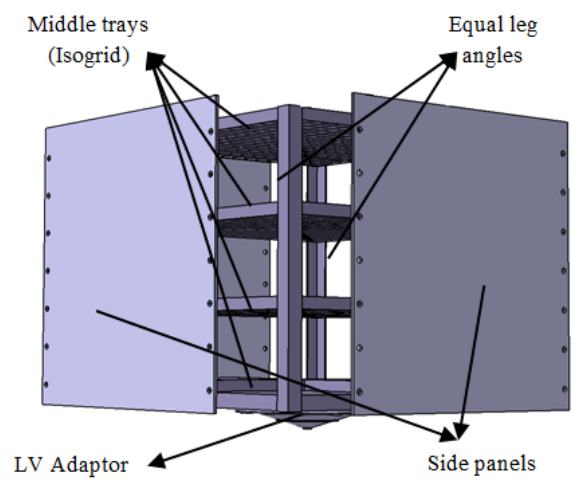

Figure 1. Satellite primary structures

\section{B. Subsystems mass budget}

For different equipments accommodations, four middle trays including the bottom and top trays are considered. The mass budgets of different subsystems including Attitude Determination and Control, Command and Date Handling, Electrical Power, Thermal Control and Telecommunication have been assumed based on design estimation relationships from different sources [13][14][15][16] and is presented in table 1 .

Table 1. Satellite subsystems mass budget

\begin{tabular}{|l|l|}
\hline \multicolumn{1}{|c|}{ Subsystems } & \multicolumn{1}{c|}{ Mass budget } \\
\hline Attitude Determination and Control & $10 \%$ \\
\hline Command and Data Handling & $5 \%$ \\
\hline Power and Cabling & $23 \%$ \\
\hline Telecommunication & $5 \%$ \\
\hline Thermal control & $2 \%$ \\
\hline Structure and Payload & To be calculated \\
\hline
\end{tabular}

In this work, the payload mass budget and primary structures mass budget are not pre-assumed although their total mass budgets should not exceed than $55 \%$ of the satellite total mass. In this way, as much as reduction in structure mass resulted from different considerations, there will be more mass budget available for the payload. This approach has special advantageous in university microsatellite projects which at the early stages of the design there is no exact information about the payload.

\section{STRUCTURE MODELING}

For structural sizing the satellite is modeled in such a way that provides capability of rapid static and dynamic analysis. In static part the calculations are done on each set of primary structures. The satellite bottom tray assumed to be clamped with the launch vehicle. Maximum stress, deflection and buckling of each primary structure element are analyzed. In dynamic modeling, a simple mass-spring model is used in order to obtain a rough estimation of satellite natural frequency in both longitudinal and lateral directions. Structure modeling is done based on analytical design formulas from classic structure design references [17][18][19].

In design process the load limits induced by launch vehicle on the satellite is considered and transportation and handling loads are regarded as inferior to the values of the launch environment. Usually in university microsatellite projects it is common to use piggy back launch services. It means that the satellite will be launched to the orbit as a secondary payload of the launch vehicle. This strategy will decrease the launch cost which is an important portion of the whole project expenses. Considering this and uncertainties about final launch vehicle at the early stages of the design, the Arian Structure for Auxiliary Payload 5, ASAP 5, [20] requirements is selected as the basement of structural design. The strength and stiffness requirements applied to the structural design are indicated in table 2 .

Table 2. Structural requirements from ASAP5 [20]

\begin{tabular}{|l|l|l|l|}
\hline Requirement & \multicolumn{3}{|c|}{ Description } \\
\hline \multirow{2}{*}{ Strength } & Design limit loads & Longitudinal & Lateral \\
\cline { 2 - 4 } & Acceleration (g) & $-7.5 \mathrm{~g} /+5.5 \mathrm{~g}$ & $\pm 6 \mathrm{~g}$ \\
\hline \multirow{2}{*}{ Stiffness } & Fundamental freq. (longitudinal) & $\geq 90 \mathrm{~Hz}$ \\
\cline { 2 - 4 } & Fundamental freq. (lateral) & $\geq 45 \mathrm{~Hz}$ \\
\hline
\end{tabular}

Also, the requirements from table 1, are applied in structural analysis by safety factor of 1.5 and uncertainty factor of 1.5 .

\section{A. Static model}

For static analysis, satellite is considered to be clamped at the attached point to the launch vehicle, Fig.2. Based on the flight limit loads of the launch vehicle and considering the design factors the design load is calculated. Uniform load distribution is assumed in lateral direction. Maximum normal stress from direct longitudinal force and lateral force bending moment and maximum shear stress are calculated. Buckling analysis is done for each of primary structure elements separately. Buckling of equal leg angle beams is determined. Buckling of thin plates may occur from compression, shear, 
bending and also under combined stress situations. In satellite model, for each pair of in front side panels two cases of buckling is assumed. First, buckling just from compression stress and second, buckling from combined stress; bending and shear.

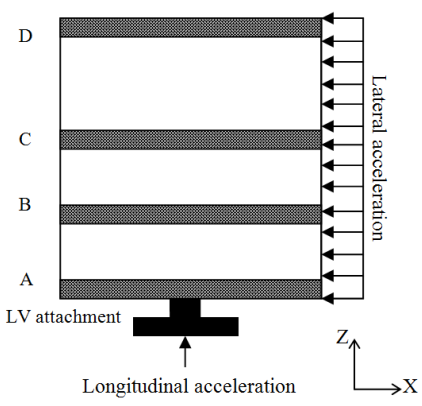

Figure 2. Satellite static model

\section{B. Dynamic model}

For stiffness requirement evaluation, four degrees of freedom mass-spring system is considered for obtaining rough natural frequency of the satellite. As seen in Fig. 3, each degree of freedom in longitudinal direction is representative of satellite trays displacement with respect to the satellite attachment plane to launch vehicle. And for lateral direction, as seen in Fig. 4, there are three degrees of freedom related to satellite trays movement in lateral direction and one rotational degree of freedom for satellite angular displacement in its attachment point with launch vehicle.

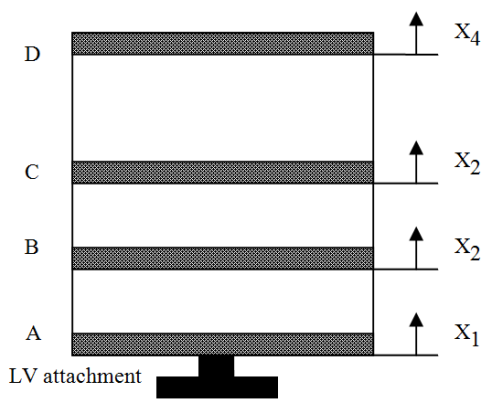

Figure 3. Satellite dynamic model in longitudinal direction

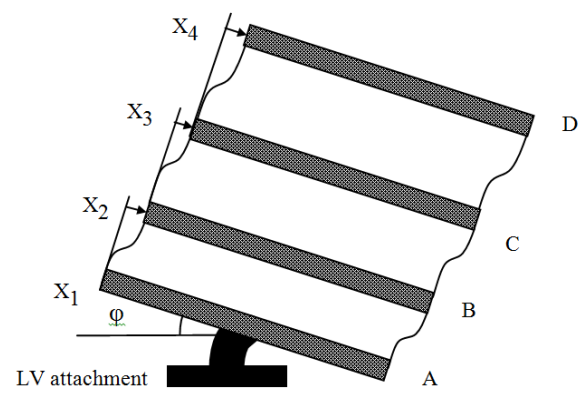

Figure 4. Satellite dynamic model in lateral direction

Considering the four degrees of freedom mass-spring system motion equations, satellite mass distribution on each tray and equivalent stiffness of primary structure elements, an eigenvalue problem is established. By solving this eigenvalue problem for each of longitudinal and lateral directions, satellite minimum natural frequencies are calculated.

\section{STRUCTURAL SIZING TOOL}

Based on satellite structural model described in section III, different parts of calculation are linked together in MATLAB environment to form a unified sizing tool which its process consists of following main steps:

- Creating the primary structure elements database in a practical range of variation for their geometrical parameters.

- Introducing an initial mass, $m_{i}$, enabling the program to calculate the launch vehicle loads exerted on the satellite.

- Conducting static calculations for maximum stress, maximum deflection and buckling for each set of primary structures.

- Selecting the acceptable primary structure set based on positive margin of safety and minimum mass criteria.

- Recalculating satellite total mass based on calculated structural mass.

- Correcting the initial introduced mass as following:

a) If the calculated satellite total mass is more than $m_{i}$, the payload mass budget is decreased in such a way that both structure mass and payload mass budgets fulfill the condition of being $55 \%$ of satellite total mass.

b) If the calculated satellite total mass is less than $m_{i}$, this mass difference is added to the payload mass budget fulfilling the condition of structural and payload mass budgets being $55 \%$ of satellite total mass.

- Determination of mass and stiffness matrices according to the corrected mass distribution.

- Solving eigenvalue problem in order to find minimum natural frequency in both lateral and longitudinal directions.

- Repeating all calculations with a different set of primary structures in case of having minimum natural frequency less than required.

\section{A. Design variables}

Design variables consist of three primary structure elements geometrical properties as well as three system level variables. The first part includes the thickness of side panels and thickness and leg length of equal leg angle beams. The system level variables are satellite total mass and its dimensions in lateral $(\mathrm{X} \& \mathrm{Y})$ and longitudinal $(\mathrm{Z})$ directions. According to different launch vehicles requirements regarding volume available for secondary payloads, and also common machining tools available at universities mechanical workshops, the range of practical variations of these variables are selected as indicated in table 3. 
Table 3. Structural sizing tool design variables

\begin{tabular}{|c|c|c|c|c|c|c|}
\hline Variables & $\begin{array}{c}m_{\text {sat }} \\
{[\mathrm{kg}]}\end{array}$ & $\begin{array}{c}t \\
{[\mathrm{~mm}]}\end{array}$ & $\begin{array}{c}b \\
{[\mathrm{~mm}]}\end{array}$ & $\begin{array}{c}t_{\text {bar }} \\
{[\mathrm{mm}]}\end{array}$ & $\begin{array}{c}l_{X Y} \\
{[\mathrm{~m}]}\end{array}$ & $\begin{array}{c}l_{Z} \\
{[\mathrm{~m}]}\end{array}$ \\
\hline $\begin{array}{c}\text { Variation } \\
\text { boundaries }\end{array}$ & {$[20,70]$} & {$[1,5]$} & {$[20,30]$} & {$[1,5]$} & {$[0.40,0.50]$} & {$[0.50,0.60]$} \\
\hline
\end{tabular}

In table $3, m_{\text {sat }}$ is the satellite total mass, $t$ is the side panel thickness, $b$ is equal leg angle beam leg length, $t_{b a r}$ is equal leg angles beam leg thickness, $l_{X Y}$ satellite dimension in lateral (X\&Y) directions, and $l_{Z}$ is satellite dimension in longitudinal (Z).

\section{RESUlts}

After checking the results of sizing tool in the defined design space search the effect of system design variables, $m_{\text {sat }}$, $l_{X Y}$ and $l_{Z}$ is evaluated and are presented in Fig. 5 to Fig. 8.

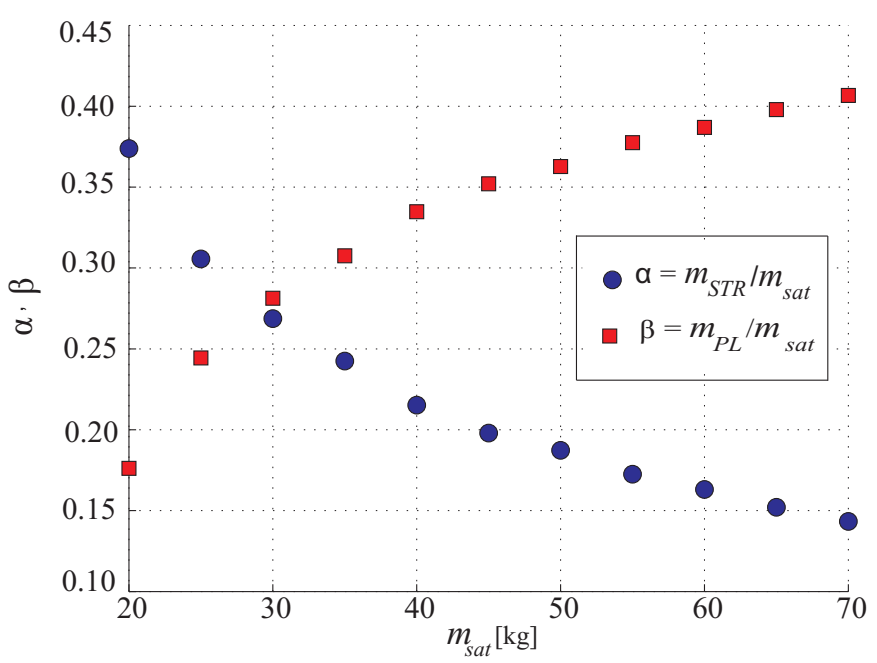

Figure 5. Satellite total mass, $m_{\text {sat }}$, effect on structure mass budget, $\alpha$, and available mass budget for payload, $\beta$, when $l_{X Y}=0.40 \mathrm{~m}$ and $l_{Z}=0.50 \mathrm{~m}$

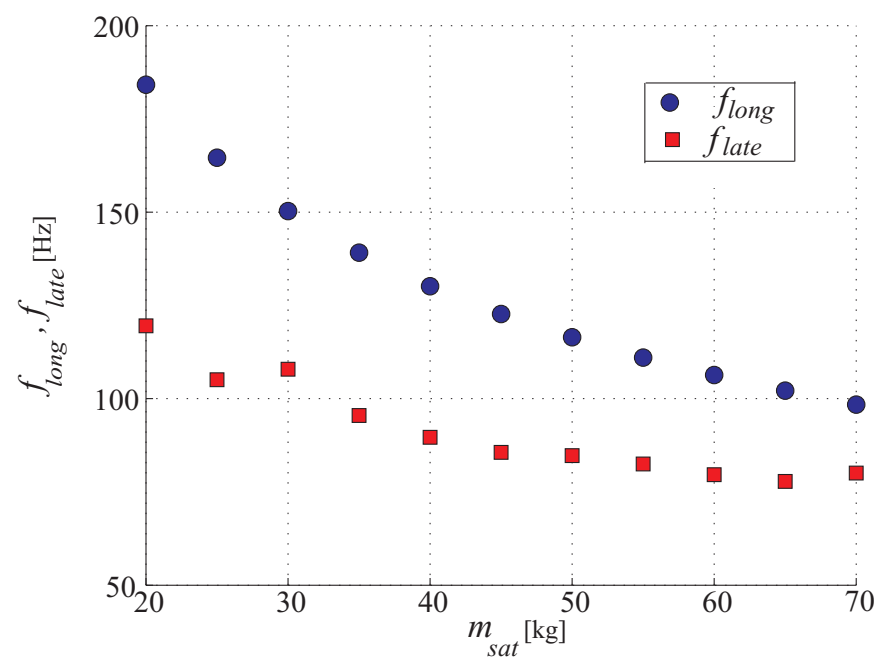

Figure 6. Satellite total mass, $m_{\text {sat }}$, effect on satellite natural frequencies, $f_{\text {long }}$ and $f_{\text {late }}$, when $l_{X Y}=0.40 \mathrm{~m}$ and $l_{Z}=0.50 \mathrm{~m}$
In Fig. 5, it is seen that by increasing the satellite total mass, the structure mass budget decreases. This results to more available mass budget for the payload. And, Fig. 6 shows that by increasing the satellite total mass while keeping the satellite dimensions constant, the natural frequency will decrease in both longitudinal and lateral directions.

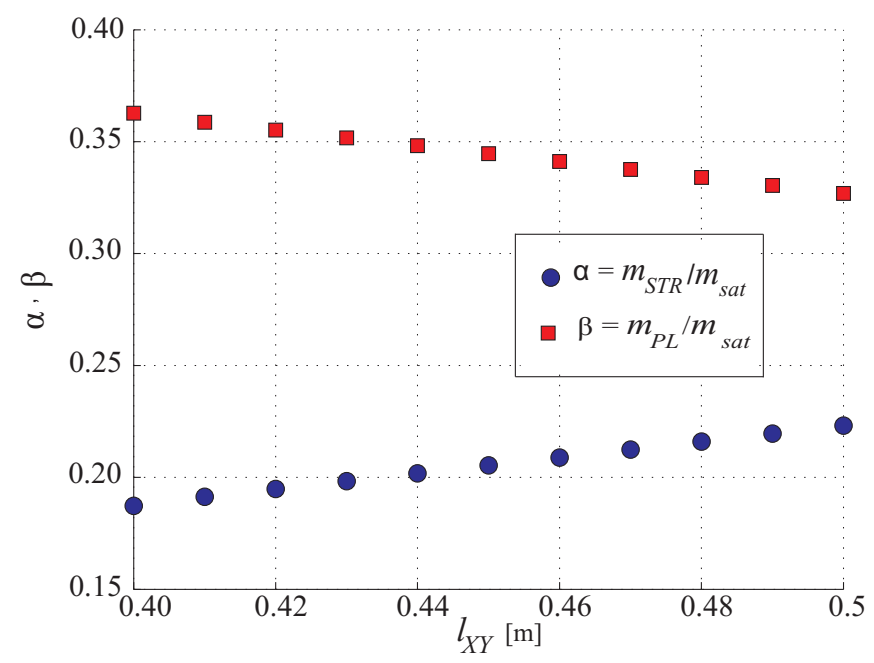

Figure 7. Satellite dimension in XY direction, $l_{X Y}$, effect on structure mass budget, $\alpha$, and available mass budget for payload, $\beta$, when $m_{\text {sat }}=50 \mathrm{~kg}$ and $l_{Z}=0.50 \mathrm{~m}$

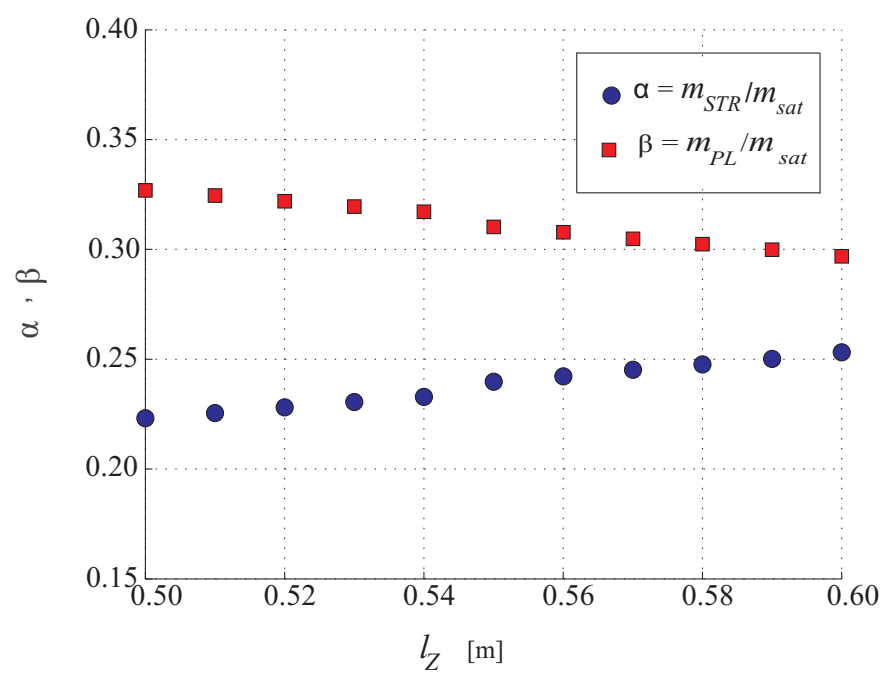

Figure 8. Satellite dimension in $\mathrm{Z}$ direction, $l_{Z}$, effect on structure mass budget, $\alpha$, and available mass budget for payload, $\beta$, when $m_{s a t}=50 \mathrm{~kg}$ and $l_{X Y}=0.50 \mathrm{~m}$

In Fig. 7 and Fig. 8, it is seen that by increasing the dimensions of the satellite the structure mass budget will increase. This increment in structure mass is less than $5 \%$ while the satellite side surfaces will increase $10 \mathrm{~cm}$ in case of Fig. 7 which will cause $36 \%$ increment in solar array surface in case of body mounted solar array assumption. This can result to considerable increment electrical power production from system engineering point of view. 


\section{CONCLUSIONS}

Satellite design at any class is a complex, iterative process. In university class microsatellite projects this inherent complexity is accompanied by constraint budgets and special requirements regarding the size and mass budget of the satellite. Developing rapid and simple sizing tools for different subsystems containing major system effects on the whole satellite design process is really useful for the design team in order to evaluate an acceptable design space for different parameters. Structure subsystem can be considered as the most affected subsystem by launch vehicle requirements in small low cost satellite projects in which usually the satellite is intended to be launched as piggy back payload. On the other hand, the concept of material and structural elements can affect the total cost of the project. Due to these facts, a rapid and simple structural sizing tool developed in order to evaluate a practical design space before design decision about mass and dimensions of the satellite. The results seems promising for the first stages of the design while the sizing tool covers an extended design space specially relating to the dimension and mass range of such projects.

\section{FUTURE WORKS}

Satellite dimensions can highly affect the electrical power subsystem regarding available area of solar arrays. Electrical power sizing tool can be developed and linked to the actual sizing tool to obtain different tradeoffs. As well more satellite architectures and primary structure elements can be considered in structural sizing tools. In this case more design variables are available and the design space is more comprehensive. After sizing tool completion and validation an optimization algorithm can be linked to the design space search for looking for optimum design point(s).

\section{REFERENCES}

[1] Sweeting, M. N., "UoSAT-1: A Review of Orbital Operations and Results", Journal of the Institution of Electronic and Radio Engineers, Volume 57, Number 5, Pages S184-S194, 1987.

[2] Swartwout, M., "Twenty (plus) years of university-class spacecraft, a review of what was, an understanding of what is, and a look at what should be next", Proceedings of $20^{\text {th }}$ Annual AIAA/USU Conference on Small Satellites, 2006, USA.

[3] Marjoniemi, K., Syvanen, L., Hoffren, M., Langlois, S., "Modular structure for small satellites", Proceedings of 4S Symposium on Small Satellites, Systems and Services (ESA SP-571), 2004, France.

[4] Sanz-Andrez, A., Meseguer, J., Perales, J. M., and Santiago-Prowald, J., "A small platform for astrophysical research base don the UPM-SAT 1 satellite of the Universidad Politécnica de Madrid", Journal of Advances in Space Research, Volume 31, Issue 2, Pages 375-380, 2003.

[5] Goldberg, H. R., and Gilchrist, B. E., "The Icarus student satellite projects”, Acta Astronautica, Volume 56, Issues 1-2, Pages 107-114, 2005.

[6] Thyagarajan, K., Gupta, J. P., Goel, P.S., and Jayaraman, K., "University small satellite program-ANUSAT", Acta Astronautica, Volume 56, Issues 1-2, Pages 89-97, 2005.

[7] Tortora, P., and Troiani, E., "The microsatellite research program at Università di Bologna", Acta Astronautica, Volume 56, Issues 7, Pages 696-704, 2005.
[8] Ravanbakhsh, A., and Franchini, S., "Preliminary Structural Sizing of a Modular Microsatellite Based on System Engineering Considerations", Proceedings of $3^{\text {rd }}$ International Conference on Multidisciplinary Design Optimization and Applications, Paris, France, June 2010.

[9] "Isogrid Design Handbook", NASA CR-124075, McDonnell Douglas Astronautics Comp., Revision A, February 1973.

[10] Wu, J.H., Liu, A.Q., and Chen, H.L., "Exact solutions for free-vibration analysis of rectangular plates using Bessel functions", Journal of Applied Mechanics, Volume 74, Issues 6, Pages 1247-1251, 2007.

[11] Quincieu, J., "Structure Design for Modular Platform and Capability on the USUsat2 Micro-satellite", Proceedings of Space 2004 conference and Exhibit, 2004, California, USA.

[12] Hillis, D., Brackley, N., Keil, T., Pernicka, H., "Structures conceptual Design", Missuri University of Science and Technology, 2009.

[13] Larson, W.J., and Wertz, J.R. "Space mission analysis and design ( $3^{\text {rd }}$ edition)", Microcosm Press and Kluwer, 1999, USA.

[14] Fortescue, P., and Stark, J., "Spacecraft systems engineering ( $3^{\text {rd }}$ edition)", John Wiley and Sons Inc, 2003, USA.

[15] Brown, C.D., "Elements of spacecraft design", AIAA Education Series, 2002, USA.

[16] Chang, Y.K., Hwang, K.L., and Kang, S.J., "SEDT (System Engineering Design Tool) development and its application to small satellite conceptual design", Acta Astronautica, Volume 61, Issues 7-8, Pages 676-690.

[17] Bruhn, E.F., "Analysis and design of flight vehicle structures", Jacobs Publishing Inc, 1973, USA.

[18] Thomson, W.T., and Dahleh, M.D., "Theory of vibration with application", Prentice Hall, 1998,USA.

[19] Sarafin, T. P., "Spacecraft structures and mechanisms", Space Technology Library, 2003, USA.

[20] "Ariane Structure for Auxiliary Payload 5 User's Manual", Ariane space, 2000. 